\title{
Occurrence of L-iduronic acid and putative D-glucuronyl C5-epimerases in prokaryotes
}

\author{
John Raedts • Servé W. M. Kengen • John van der Oost
}

Received: 13 December 2010/Revised: 14 January 2011 /Accepted: 18 January 2011 /Published online: 24 February 2011

(C) The Author(s) 2011. This article is published with open access at Springerlink.com

\begin{abstract}
Glycosaminoglycans (GAGs) are polysaccharides that are typically present in a wide diversity of animal tissue. Most common GAGs are well-characterized and pharmaceutical applications exist for many of these compounds, e.g. heparin and hyaluronan. In addition, also bacterial glycosaminoglycanlike structures exist. Some of these bacterial GAGs have been characterized, but until now no bacterial GAG has been found that possesses the modifications that are characteristic for many of the animal GAGs such as sulfation and C5-epimerization. Nevertheless, the latter conversion may also occur in bacterial and archaeal GAGs, as some prokaryotic polysaccharides have been demonstrated to contain L-iduronic acid. However, experimental evidence for the enzymatic synthesis of Liduronic acid in prokaryotes is as yet lacking. We therefore performed an in silico screen for D-glucuronyl C5-epimerases in prokaryotes. Multiple candidate C5-epimerases were found, suggesting that many more microorganisms are likely to exist possessing an L-iduronic acid residue as constituent of their cell wall polysaccharides.
\end{abstract}

Keywords Glycosaminoglycans · L-iduronic acid · Dglucuronyl C5-epimerase $\cdot$ Lipopolysaccharide $\cdot$ Capsule polysaccharide

\section{Introduction}

Glycosaminoglycans (GAGs) are long linear negatively charged hetero-polysaccharides, consisting of repeating

J. Raedts $(\bowtie) \cdot$ S. W. M. Kengen $\cdot$ J. van der Oost Laboratory of Microbiology, Wageningen University, Dreijenplein 10,

6703 HB Wageningen, The Netherlands

e-mail: John.Raedts@wur.nl disaccharide residues usually of a hexuronic acid linked to a hexosamine. Glycosaminoglycans are ubiquitously found throughout the animal kingdom, where they are involved in a wide variety of biological processes. The most common glycosaminoglycans are chondroitin sulfate, dermatan sulfate, heparan sulfate, heparin, hyaluronan and keratan sulfate. An overview is given in Table 1.

The glycosaminoglycan hexuronic acid residue is either D-glucuronic acid or its C5 epimer L-iduronic acid. The latter is a rather unique hexuronic acid that is typically found in glycosaminoglycans, while D-glucuronic acid is a very common compound in nature. The epimerization of $\mathrm{D}$ glucuronic acid towards L-iduronic acid is mediated by a D-glucuronyl C5-epimerase acting at a polysaccharide level after D-glucuronic acid incorporation [1, 2]. The C5epimerization of D-glucuronic acid is essential for specific binding properties of versatile glycosaminoglycans like heparin and heparan sulfate. Another D-glucuronyl C5epimerase does exist responsible for dermatan sulfate biosynthesis, as well as a $\mathrm{C} 5$-epimerase involved in alginate biosynthesis. These epimerases do not show sequence homology to the heparin D-glucuronyl C5-epimerase [3].

Glycosaminoglycans traditionally are isolated from animal tissue. A major drawback is the great polydispersity of animal-derived material, both in chain length and degree of epimerization/sulfation. Large scale chemical synthesis of heparin is not feasible, because of the $\mathrm{C} 5$-epimerization of D-glucuronic acid being one of the major bottlenecks. Liduronic acid can be synthesized chemically, however C5epimerization of D-glucuronic acid as part of a polymer only can be done enzymatically. In the past decades the C5epimerase in animal heparin biosynthesis has been identified and characterized [4], and has some major limitations to use in large scale biotechnological production processes. Novel D-glucuronyl C5-epimerases that have less restric- 
Table 1 Overview of the most common glycosaminoglycans. GlcA, glucuronic acid; GalNAc, $N$-acetyl-galactosamine; GlcNAc, $N$-acetylglucosamine; IdoA, iduronic acid; GlcNS, $N$-sulfate-glucosamine; Gal, galactose; C\#, carbon number; ECM, extracellular matrix

\begin{tabular}{llll}
\hline Glycosaminoglycan & Main disaccharide & Sulfation pattern & Localization \\
\hline Chondroitin sulfate & $-4)$ GlcA $\beta(1-3)$ GalNAc- $\beta(1-$ & GalNAc at C4/C6 and GlcA at C2 & cartilage, bone, heart valves \\
Dermatan sulfate & $-4) \operatorname{IdoA} \alpha(1-3)$ GalNAc- $\beta(1-$ & GalNAc at C4/C6 and IdoA at C2 & skin, blood vessels, heart valves \\
Heparan sulfate & $-4) G l c A \beta(1-4)$ GlcNAc- $\alpha(1-$ & GlcA(IdoA) at C2, GlcNAc(NS) at C6, C3 & cell surface membranes, ECM \\
Heparin & $-4) \operatorname{IdoA} \alpha(1-4)$ GlcNS- $\alpha(1-$ & like heparan sulfate but heavier & mast cells $(e . g$. lungs, liver, skin) \\
Hyaluronan & $-4) \mathrm{GlcA} \beta(1-3)$ GlcNAc- $\beta(1-$ & completely unsulfated & synovial fluid, eye, ECM, skin \\
Keratan sulfate & $-3)$ Gal $\beta(1-4) G l c N A c-\beta(1-$ & Gal and GlcNAc at C6 & cornea, bone, cartilage \\
\hline
\end{tabular}

tions in substrate acceptance, better stability and easier production methods would have great potential in controlled chemo-enzymatic synthesis of L-iduronic acid containing polymers like heparin and heparin-analogs. In this review we discuss putative novel C5-epimerases that might convert D-glucuronic acid to L-iduronic acid with less restrictions.

\section{Glycosaminoglycan-like structures in bacterial capsules}

Traditionally, GAGs were considered as polymeric structures only to be found in (in)vertebrate animals. However, in the past decades, some polyanionic bacterial cell wall polysaccharides have been described, of which the structure has resemblance to some well-characterized animal glycosaminoglycans. Microorganisms possessing GAGs generally are pathogenic bacteria in which the surface exposed capsular polysaccharides are likely to serve as a virulence factor. The resemblance between the bacterial capsule and the animal GAG results in very limited or no response of the hosts' immune system. GAG-containing pathogens, including serotypes of both Escherichia coli and Pasteurella multocida, are discussed below.

E. coli GAGs Many different serotypes of $E$. coli have been described. Discrimination between these strains is generally based on antigenic studies. Mostly differences can be found in a specific part of the bacterial lipopolysaccharide (LPS), the so-called $\mathrm{O}$-antigen. To a lesser extent differences are seen in antigenic properties of the flagella (H-antigen) and the bacterial capsule (K-antigen). The latter is a protective layer of polysaccharides that generally can be easily observed by light microscopy. Over 70 different Kantigens have been described [5]. As discussed below, two of them are analogous to glycosaminoglycans as found in animals.

A chondroitin-like glycosaminoglycan has been isolated from E. coli $\mathrm{O} 5: \mathrm{K} 4: \mathrm{H} 4$ [6]. Identical to chondroitin this "K4 capsular polysaccharide" consists of equimolar amounts of $\beta$-D-glucuronic acid and $\beta$ - $N$-acetyl-galactosamine. Unlike chondroitin an additional fructose is $\beta(1-3)$ linked as a substituent to each GlcA residue. However, this modification can easily be removed by mild acidification, resulting in a chondroitin backbone. Upon removal of the fructose residue, the immune response against the $\mathrm{K} 4$ polysaccharide decreases considerably.

Another glycosaminoglycan-like structure has been described for the K5 antigen of E. coli O10 : K5 : H4. This capsular polysaccharide has an identical structure to heparosan, the unsulfated and non-epimerized backbone structure of heparan sulfate and heparin. The $\mathrm{K} 5$ capsule is a linear polysaccharide containing $\alpha-N$-acetyl-glucosamine and $\beta$-glucuronic acid in equimolar amounts, linked by an (1-4) glycosidic bond [7]. In contrast to animals, no postpolymerization modifications occur on the heparosan molecule. This makes the K5 polysaccharide a useful substrate to study the enzymes in heparin biosynthesis $[8$, 9], and a potential precursor for chemo-enzymatic synthesis of heparin.

P. multocida GAGs Similar glycosaminoglycans have been isolated from several serotypes of another pathogenic gammaproteobacterium, namely Pasteurella multocida. The capsules of $P$. multocida type A, D and F could be removed upon treatment with different glycosaminoglycan hydrolases [10]. A more detailed analysis of the capsular polysaccharides of P. multocida type $\mathrm{D}$ and $\mathrm{F}$ has revealed similarity with the $\mathrm{K}$ antigens of E. coli $\mathrm{K} 5$ and $\mathrm{K} 4$ respectively. The P. multocida type $\mathrm{D}$ polymer is identical to heparosan, the type $\mathrm{F}$ polymer is unmodified chondroitin [11].

Additionally, another P. multocida capsular polysaccharide has been described to be analogous to a vertebrate glycosaminoglycan. The extracellular capsule of $P$. multocida type A is chemically identical to the animal GAG hyaluronan [12]. In addition, multiple species of Streptococci have been described to have such a hyaluronan capsule. All these strains are pathogenic to human or other mammals, the hyaluronan capsule having an important role in preventing an immune response. Since these molecules are identical to 
mammalian hyaluronan, bacterially produced hyaluronian has substantial commercial value. In addition to animal derived hyaluronan, it is nowadays widely commercially available for numerous existing applications.

Although multiple examples of bacterial GAGs do exist, for none of these glycosaminoglycans modifications have been observed similar to those in the GAG biosynthesis pathways in animals. A key modification step in these pathways is the C5-epimerization of D-glucuronic acid towards L-iduronic acid, catalyzed by a D-glucuronyl C5epimerase. No bacterial counterpart of this enzyme has been experimentally characterized to date. However, the presence of L-iduronic acid in extracellular polysaccharides of several microorganisms, suggests that D-glucuronyl C5epimerases do exist in prokaryotes.

\section{Identification of iduronic acid in microbes}

While being a well-known component of (animal) glycosaminoglycans, the presence of L-iduronic acid in prokaryotes is rather uncommon. For some time it was believed that L-iduronic acid could only be found in multicellular eukaryotes. However, as discussed below, in the last decades multiple examples of microbial L-iduronic acid have been published.

\section{Bacteria}

The first case of L-iduronic acid being present in a prokaryote was reported in a study of the gram-positive bacterium Clostridium perfringens NCTC 10578 [13]. L-iduronic acid was identified in a purified "type-specific" polysaccharide from Clostridium perfringens strain Hobbs 10. The exact polysaccharide structure is still unknown but the L-iduronic acid level in the isolated polysaccharide is estimated to be $7 \%$. Most likely this "type-specific" polysaccharide is part of a bacterial capsular polysaccharide [14].

Another report on the presence of L-iduronic acid in a prokaryote concerns the analysis of specific extracellular polysaccharide (EPS) of Butyrivibrio fibrisolvens strain X6C61 [15]. As much as 37 strains of B. fibrisolvens were screened in total, but only a single strain appeared to contain L-iduronic acid. This indicates that L-iduronic acid is part of a type-specific EPS. The exact composition of the EPS remains to be characterized, although it has been proposed that L-iduronic acid is associated to a galactosamine residue.

In addition, several reports exist in which L-iduronic acid is identified as a compound of an O-specific polysaccharide. An O-antigen is the highly variable part of a lipopolysaccharide (LPS), which is present in the outer membrane of gram-negative bacteria. The first report of Liduronic acid being present in an $\mathrm{O}$-antigen was after structure elucidation of the O-antigen of the marine bacterium Pseudoalteromonas haloplanktis strain KMM 223 (44-1) [16]. The L-iduronic acid residue is part of a pentasaccharide (Fig. 1) that additionally contains two Dglucuronic acid residues and two residues of the uncommon QuiN4N (2,4-diamino-2,4,6-trideoxyglucose). The high amount of hexuronic acids results in a highly acidic Oantigen. In addition, the uncommon QuiN4N residues and GlcA residues have been found in other serotypes of Pseudoalteromonas [17], however strain KMM 223 remains the only example that has an L-iduronic acidcontaining O-antigen.

More recently, two additional O-antigens have been identified in which L-iduronic acid is one of the building blocks (Fig. 1). Both Escherichia coli type 112ab and Shigella boydii B15, have an identical pentasaccharide structure [18, 19]. Many more O-antigen structures of various serotypes of both $E$. coli and $S$. boydii have been resolved to date, however L-iduronic acid seems to be restricted to these two reported strains. Just like the previous reports dealing with L-iduronic acid identification in bacteria, the occurrence of this structure is highly typespecific rather than a general feature.

\section{Archaea}

L-iduronic acid has been reported in archaea only once. There is evidence of the presence of iduronic acid in a cell surface lipoprotein of Halobacterium halobium [20]. The cell wall of this archaeon is a glycoprotein based S-layer. The glycoprotein has two specific forms of $\mathrm{N}$-glycosylation. First each polypeptide consists of a single glycosaminoglycan-like polysaccharide with a [-4)GalNAc(1-4)GalA(1-3)-GalNAc (1- $]_{\mathrm{n} 10-15}$ backbone attached. Apart from that, there are 12 potential glycosylation sites where an Asn-Glc (asparaginylglucose) linkage unit is extended by two or three $\beta(1-4)$ bound glucuronic acid residues. About $1 / 3$ of these glucuronic acid moieties are replaced by an iduronic acid (Fig. 1). An identical glycoconjugate can be found at the organisms' flagellin [21]. Although archaeal flagellins often undergo posttranslational modification [22], until now this is the only report of iduronic acid presence in such a structure.

Non-canonical L-iduronic acid containing polymers in eukaryotes

L-iduronic acid traditionally is considered to be a component in many common animal glycosaminoglycans. Apart from these well-characterized GAGs, recently L-iduronic acid also has been identified in some atypical polymers. 


\section{Pseudoalteromonas \\ Haloplanktis KMM 223 (44-1)}

$(44-1)$

\author{
$\rightarrow 4)$ - $\beta$-D-GlcA-( $1 \rightarrow 4)-\beta-D-G l c A-(1 \rightarrow 3)-\beta-D-Q u i N H b 4 N H b-(1 \rightarrow 2)-\alpha-L-I d o A-(\rightarrow$ \\ 4 \\ $\uparrow$ \\ a-D-QuiNAc4NAc
}

Hanniffy et al., 1998

\section{Escherichia coli 0112ab \& Shigella boydii B15}

$\beta-D-G I c N A c$

$$
\begin{aligned}
& 1 \\
& \downarrow \\
& 3
\end{aligned}
$$

$1 / 3$ of GlcA residues can be replaced by IdoA

Wieland et al., 1985
Fig. 1 Known prokaryotic structures containing L-iduronic acid; bacterial $\mathrm{O}$-antigens and the Halobacterium halobium glycoconjugate. GlcA, glucuronic acid; QuiNHb4N, 2,4-diamino-2,4,6-trideoxy-D- glucose (Hb, S-3-hydroxybutyryl; Ac, acetyl); IdoA, iduronic acid; GlcNAc, $N$-acetyl-glucosamine; GalNAc, $N$-acetyl-galactosamine
The eukaryotic organisms having these non-canonical polymers usually do not possess the traditional GAGs as found in animals. Possibly the formation of L-iduronic acid is the result of another C5-epimerase than the heparosan Dglucuronyl C5-epimerase. An overview of some of these rare L-iduronic acid containing structures is provided below.

Algae Pleurochrysis haptonemofera is a unicellular coccolithophorid marine alga. It produces coccolith, a calcified scale. Apart from carbonate crystals, this scale contains a small amount of polysaccharide called "coccolith matrix acidic polysaccharide" (CMAP). The structure of CMAP has been determined to be composed of a repeating disaccharide structure, of which L-iduronic acid is one of the sugars [23]. In addition there are reports of the existence of L-iduronic acid in specific polysaccharides in multicellular algae. The cell wall of sea lettuce (genus Ulva) includes four types of polysaccharides, of which the watersoluble ulvan is exclusively found in members of the Ulvales. This polysaccharide has a repetitive disaccharide of L-iduronic acid that is $\alpha(1-4)$ linked to a C3-sulfated rhamnose [24].

Fungi Phallic acids are specific glycuronans that can be found in the fruiting-bodies of members of the taxon Phallales. Tsuchihashi and colleagues have described the isolation of phallic acid of at least ten species, all containing L-iduronic acid. [25]. The exact structural composition is still unknown, but it has been reported that these polysaccharides are composed of $\beta$-glucuronic acid and $\alpha$-iduronic acid residues that have an (1-4) linkage. The internal ratio of these two hexuronic acids varies around $2: 1$ to $3: 1$. The polysaccharide is called protuberic acid when the ratio glucuronic acid to iduronic acid is equal to $2: 1$ [26].

Sponges Citronamides A and B are unique products that have been isolated from the Australian sponge Citronia astra. Both are non-canonical tetrapeptides with a linked 3or 4-O-(aminocarbonyl)- $\alpha$-iduronic acid residue. These compounds accidently have been co-isolated with Dysinosin A, a potential serine protease (thrombin) inhibitor. Citronamides $\mathrm{A}$ and $\mathrm{B}$ are structurally not related to Dysinosin A, and the biological function of these products still needs to be clarified [27].

\section{Identification of C5-epimerases in prokaryotes}

The above described polymeric structures are examples of L-iduronic acid containing polysaccharides and glycosaminoglycan-like structures in several microorganisms. The existence of L-iduronic acid does suggest Dglucuronyl C5-epimerase activity to occur in these organisms. The wide diversity of these GAG-like structures suggests the presence of candidate $\mathrm{C} 5$-epimerases with a different or broader substrate specificity. To date no such candidate enzyme has been identified. 
In an attempt to identify candidate C5-epimerases, we screened all available prokaryotic genomes for sequences homologous to human D-glucuronyl C5-epimerase by Blast analysis (http://blast.ncbi.nlm.nih.gov/Blast.cgi) [28]. Multiple prokaryotic sequences were identified that have homology to the human sequence. Also Blasts on metagenome data reveal multiple putative prokaryotic C5epimerases. All found candidate $\mathrm{C} 5$-epimerases have a well-conserved domain making them members of the pfam06662 superfamily [29] containing the consensus of the C-terminus of D-glucuronyl C5-epimerases. An overview of all prokaryotes having one or more candidate Dglucuronyl C5-epimerase gene(s) is provided in Table 2.

The occurrences of these candidate C5-epimerases seem to be type-specific rather than species-specific. This is in line with the earlier discussed reports on the identification of L-iduronic acid containing polymers in prokaryotes, that also appear to be type-specific in various species. Among the identified candidate $\mathrm{C} 5$-epimerases differences in size exist; however, this is mainly a result of variation of the $\mathrm{N}$ terminal domain of the protein. Most prokaryotic sequences show a similar organization as the protein sequence of animal D-glucuronyl C5-epimerases. An N-terminal signal peptide is predicted for many sequences and at the $\mathrm{C}$ terminus of the protein the conserved pfam06662 domain can be found. This architecture resembles that of animal Dglucuronyl C5-epimerases. A multiple sequence alignment of the C-terminal domain of the prokaryotic candidate C5epimerase and a selection of animal C5-epimerases is included (Fig. 2). It is tempting to speculate on the role of those amino acid residues that are completely conserved. Residues possibly involved in catalysis are the conserved tyrosines and histidines. Structural data of two other types of C5-epimerases, not homologous to the heparin C5epimerase [3] and the prokaryotic candidate epimerases, reveal a role of conserved histidines and tyrosines in catalysis for both functionally distinct C5-epimerases [30, 31]. Although there is no homology at amino acid level, a similar catalytic mechanism of the heparin-acting C5epimerase to these distinct $\mathrm{C} 5$-epimerases cannot be ruled out and could be feasible.

We constructed a phylogenetic tree [32] containing several eukaryotic D-glucuronyl C5-epimerases, as well as a selection of prokaryotic homologs. The multiple sequence alignment $[33,34]$ is mostly based on the C-terminus of the genes (Fig. 2). No remarkable differences were observed when constructing a tree of the full length sequences or of the C-terminus only. Phylogenetically, the prokaryotic candidate C5-epimerase sequences cluster in a domainspecific way (Fig. 3). Most deviation is observed in bacterial sequences, while archaea and eukaryotes are more alike. Obvious inter-domain substitutions can not be observed, and are not expected to have occurred recently.
Few bacterial sequences do cluster with eukaryotes and archaea but these are close to the root and bootstrap values are too low to draw any conclusions.

On an intra-domain level one could speculate on the clustering. Considering a confidence level of $70 \%$ or higher, the archaeal genes cluster in two clades. Surprisingly the two Methanothermobacter species do not cluster with the Methano(caldo)cocci. The subset of bacterial sequences gives rise to several clades, each not necessarily clustered in a class specific way. An example is seen for Thermoanaerobacter, Bacillus and Ruminococcus that do cluster with Bacteroides and not cluster with the clostridiae genes, even though they are all in the class of firmicutes. Instead the clostridiae genes cluster with an Acidobacterium. It is tempting to speculate that these deviations reflect the occurrence of variant enzymes (paralogs) with a different substrate specificity. This is expected because of the wide diversity that exists in bacterial cell wall polysaccharides. Most likely the candidate C5-epimerases are involved in the biosynthesis of type-specific polysaccharides.

The association of the putative candidate C5-epimerases with other (predicted) sugar modifying enzymes (e.g. glycosyltransferases) is clearly revealed upon neighborhood analysis of the involved prokaryotic genomes (Fig. 4). Genes in this gene cluster are likely to be involved in strain specific O-antigen production [35], since some of the sequences have homology to the $w b b$ operon [36], which is known to be involved in the O-antigen biosynthesis [37]. As LPS do not occur in gram positives, alternatively these gene clusters can also be involved in the biosynthesis of a capsular polysaccharide. This cell wall structure can occur in gram positive bacteria like $T$. tengcongensis. The exact gene function is hard to distinguish, since genes involved in polysaccharide capsule biosynthesis are sometimes embedded in the other cell wall biosynthesis related gene regions (e.g. LPS) and vice versa [38].

A remarkable similarity in genomic organization is seen for some of the putative C5-epimerases. Figure 4 shows the flanking genes up- and downstream the candidate C5epimerases of two Vibrio cholerae strains (albensis VL426 and CO845), the thermophilic bacterium Thermoanaerobacter tengcongensis MB4 and two Photorhabdus species. Both Photorhabdus luminescens TT01 and Photorhabdus asymbiotica are symbiotic pathogens of insects, although $P$. asymbiotica is occasionally found as an opportunistic pathogen of humans as well. $V$. cholera is a well-known human pathogen, causing cholera. No virulence activity is reported for T. tengcongensis [39].

For most of these prokaryotic candidate C5-epimerases, the gene is in close proximity of various sugar modifying enzymes. The analogy in gene neighborhood organization is remarkably similar for the above mentioned five bacteria. 
Table 2 Overview candidate C5-epimerases in prokaryotes

\begin{tabular}{|c|c|c|c|c|}
\hline Organism & Class & Size & Gene ID & e-value. \\
\hline \multicolumn{5}{|l|}{ Bacteria; Acidobacteria } \\
\hline Candidatus Koribacter versatilis Ellin 345 & Candidatus koribacter & 401 aa & 94970543 & $9 e-65$ \\
\hline \multicolumn{5}{|l|}{ Bacteria; Actinobacteria } \\
\hline Sanguibacter keddieii DSM 10542 & Actinobacteridae & 355 aa & 269795908 & $1 \mathrm{e}-16$ \\
\hline Conexibacter woesei DSM 14684 & Rubrobacteridae & 473 aa & 284043115 & $3 e-19$ \\
\hline Conexibacter woesei DSM 14684 & Rubrobacteridae & 489 aа & 284046741 & $1 \mathrm{e}-26$ \\
\hline \multicolumn{5}{|l|}{ Bacteria; Bacteroidetes } \\
\hline Bacteroides sp. 1_1_6 & Bacteroidia & 305 aа & 253567783 & $1 \mathrm{e}-29$ \\
\hline \multicolumn{5}{|l|}{ Bacteria; Firmicutes } \\
\hline Bacillus cereus $R 309803$ & Bacillales & 307 aа & 229164211 & $2 \mathrm{e}-40$ \\
\hline Clostridium ljungdahlii ATCC 49587 & Clostridia & 737 aa & 300854052 & $6 e-52$ \\
\hline Clostridium ljungdahlii ATCC 49587 & Clostridia & 399 aа & 300855735 & $9 \mathrm{e}-53$ \\
\hline Clostridium ljungdahlii ATCC 49587 & Clostridia & 372 aa & 300855857 & $7 e-50$ \\
\hline Clostridium kluyveri DSM 555 & Clostridia & 411 aa & 153953040 & $1 \mathrm{e}-35$ \\
\hline Clostridium kluyveri DSM 555 & Clostridia & 372 aa & 153953635 & $2 \mathrm{e}-32$ \\
\hline Clostridium kluyveri DSM 555 & Clostridia & 452 aa & 153953720 & $1 \mathrm{e}-32$ \\
\hline Clostridium kluyveri NBRC 12016 & Clostridia & 414 aa & 219853696 & $1 \mathrm{e}-35$ \\
\hline Ruminococcus sp. $18 P 13$ & Clostridia & 302 aa & 291544017 & $6 e-34$ \\
\hline Thermoanaerobacter tengcongensis $M B 4$ & Clostridia & 312 aa & 20807150 & $3 e-58$ \\
\hline \multicolumn{5}{|l|}{ Bacteria; Proteobacteria } \\
\hline Campylobacter hominis ATCC BAA-381 & Epsilonproteobacteria & 458 aa & 154149157 & $4 \mathrm{e}-34$ \\
\hline Nautilia profundicola $\mathrm{AmH}$ & Epsilonproteobacteria & 317 aa & 224373665 & $2 \mathrm{e}-38$ \\
\hline Bermanella marisrubri sp. RED65 & Gammaproteobacteria & 448 aа & 94501207 & $2 \mathrm{e}-76$ \\
\hline Escherichia coli O112ab & Gammaproteobacteria & 323 aа & 187880578 & $1 \mathrm{e}-23$ \\
\hline Shigella boydii type 15 & Gammaproteobacteria & 323 aa & 187880569 & $1 \mathrm{e}-23$ \\
\hline Photorhabdus asymbiotica & Gammaproteobacteria & 370 aа & 253991788 & $5 e-15$ \\
\hline Photorhabdus luminescens sp. laumondii TTO1 & Gammaproteobacteria & 368 aа & 37528614 & $4 \mathrm{e}-12$ \\
\hline Vibrio cholerae bv. albensis VL426 & Gammaproteobacteria & 308 aа & 229524794 & $2 \mathrm{e}-27$ \\
\hline Vibrio cholerae st. CO845 & Gammaproteobacteria & 311 aa & 295149004 & $9 \mathrm{e}-34$ \\
\hline \multicolumn{5}{|l|}{ Archaea; Korarchaeota } \\
\hline Candidatus Korarchaeum cryptofilum OPF8 & Candidatus korarchaeum & 337 aa & 170290161 & $3 e-28$ \\
\hline \multicolumn{5}{|l|}{ Archaea; Euryarchaeota } \\
\hline Methanothermobacter marburgensis st Marburg & Methanobacteria & 381 aa & 304314521 & $8 \mathrm{e}-40$ \\
\hline Methanothermobacter thermautotrophicus $\Delta H$ & Methanobacteria & 405 aa & 15678358 & $2 \mathrm{e}-59$ \\
\hline Methanocaldococcus fervens AG86 & Methanococci & 305 aa & 241911015 & $5 e-59$ \\
\hline Methanocaldococcus infernus $M E$ & Methanococci & 298 aa & 241904910 & $6 e-48$ \\
\hline Methanocaldococcus jannaschii DSM 2661 & Methanococci & 308 aa & 15669315 & $5 e-61$ \\
\hline Methanocaldococcus vulcanius M7 & Methanococci & 305 aa & 255051598 & $2 \mathrm{e}-51$ \\
\hline Methanococcus voltae A3 & Methanococci & 330 aa & 163798575 & $7 \mathrm{e}-10$ \\
\hline Thermococcus barophilus MP & Thermococci & 367 aa & 197628867 & $3 e-22$ \\
\hline Thermococcus barophilus MP & Thermococci & 511 aa & 197629016 & $3 e-33$ \\
\hline Thermococcus onnurineus NAI & Thermococci & 520 aa & 212224590 & $3 e-32$ \\
\hline Thermococcus sibiricus MM 739 & Thermococci & 371 aa & 242398189 & $2 \mathrm{e}-27$ \\
\hline Thermococcus sibiricus MM 739 & Thermococci & 512 aa & 242398190 & $9 \mathrm{e}-35$ \\
\hline
\end{tabular}


Fig. 2 Multiple sequence alignment of C-terminal part of the candidate D-glucuronyl C5-epimerases. The positions of the first and last residues of the aligned region of the corresponding candidate C5-epimerase are indicated for each sequence. Names are abbreviated and in the same order as in Fig. 3

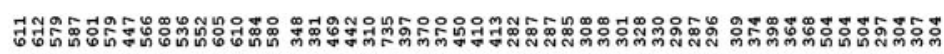

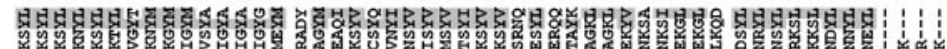

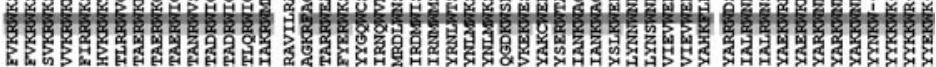
M.

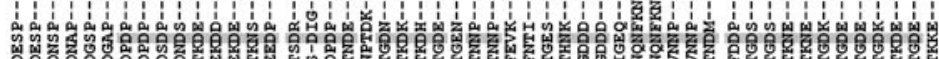
Han Tog o.

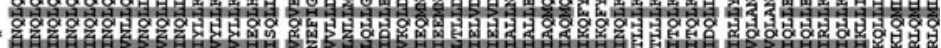

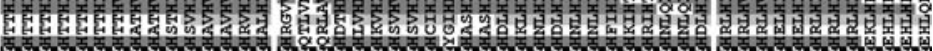

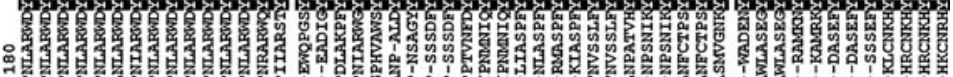

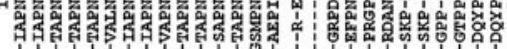

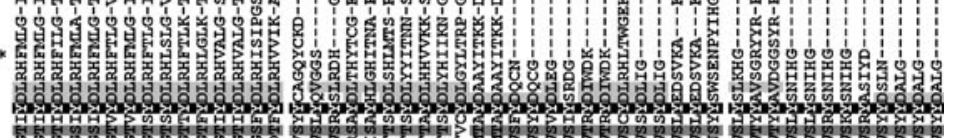
5.5. A A * ${ }_{2}$ 约

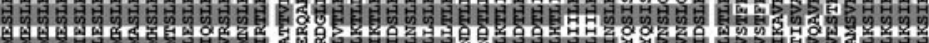

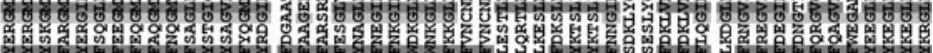

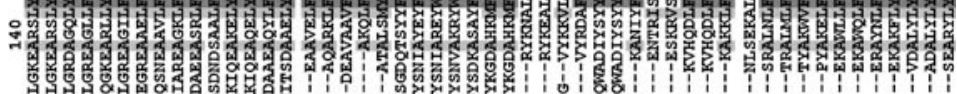
50

*

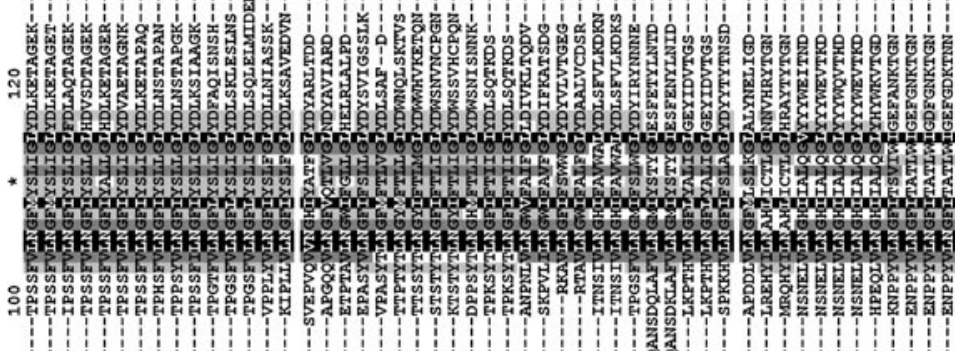
“

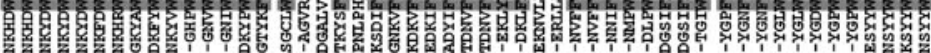
¿ 4 e 药 -

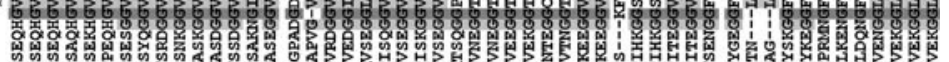
W. ๑

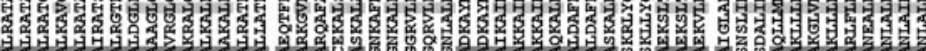
T.5.5. "Wrm

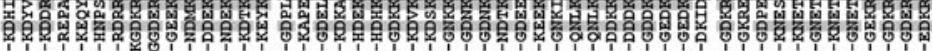

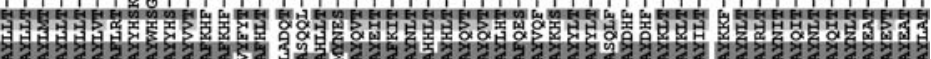

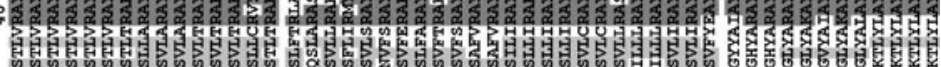

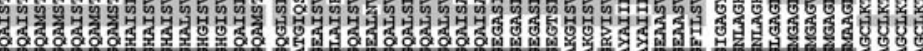
00\%0\%

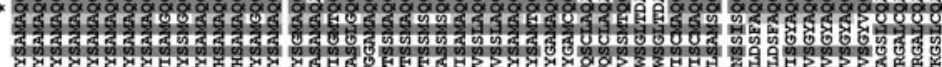

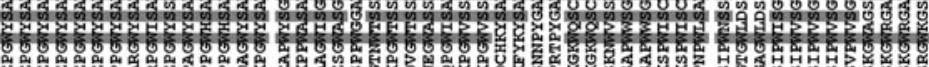

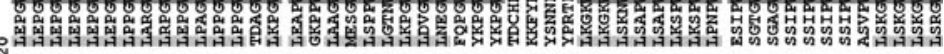

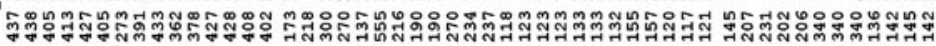

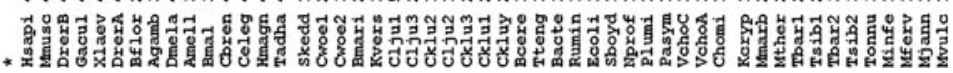




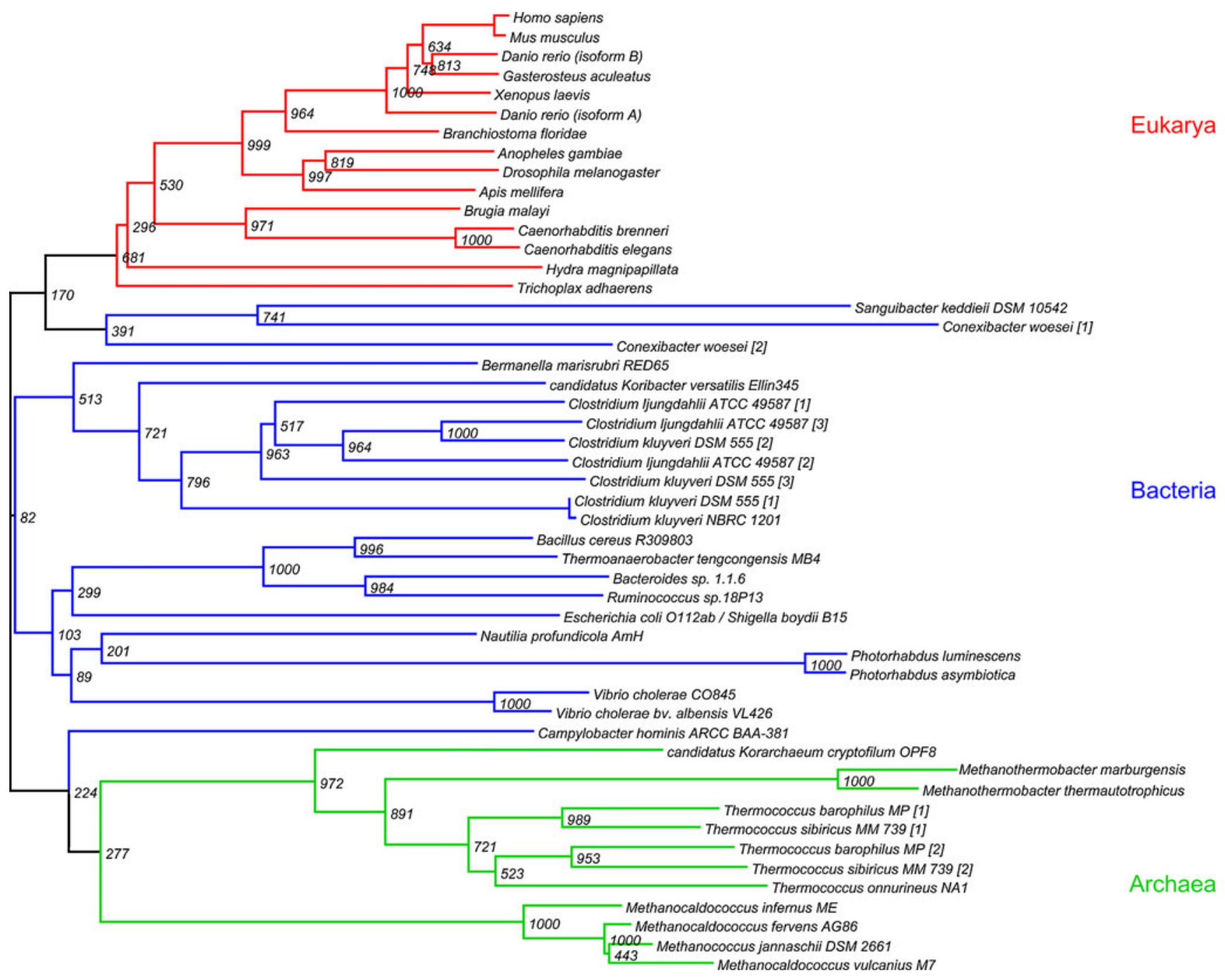

Fig. 3 Phylogenetic analysis candidate C5-epimerases. The coloring of branches is domain specific; eukarya in red, bacteria in blue and archaea in green. The used bootstrap value is 1000

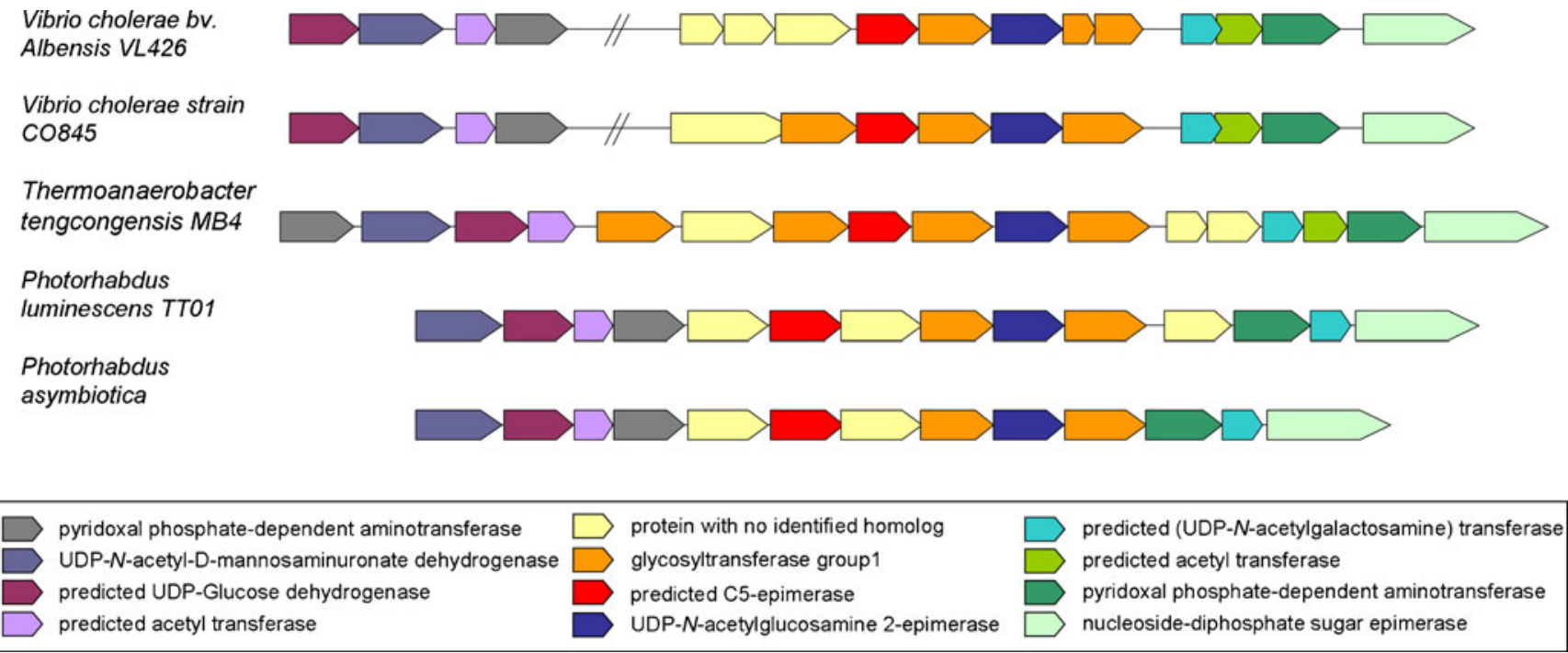

Fig. 4 Neighborhood analysis candidate C5-epimerases 
Other bacterial candidate $\mathrm{C} 5$-epimerases have a different organization of their gene neighborhood, despite the fact that they are more related to any of these five species with respect to taxonomy or candidate $\mathrm{C} 5$-epimerases sequence identity. A clear example is seen for the candidate C5epimerase of Bacillus cereus R309803. This sequence is the best hit compared to $T$. tengcongensis (54\% identity, 70\% similarity). The homology of the T. tengcongensis sequence to the putative C5-epimerases from Photorhabdus (wblE) is rather low (15\% identity, 31\% similarity). Homology with $V$. cholera is better ( $23 \%$ identity, $44 \%$ similarity), but still weak compared to the best hit.

Naturally, addition of more sequences would give a better understanding of the exact sequence distribution and a better view on the number of different clades existing. However, it is obvious that within this subset of sequences a clustering in different clades exists, which is not necessarily class-specific. An explanation of this phylogenetic distribution might be the existence of several C5-epimerase paralogs. Given the enormous variety in type-specific cell wall polysaccharides, it is expected that enzymes involved in biosynthesis have a substrate optimized specificity. Variations in substrate specificity of involved D-glucuronyl C5-epimerase would certainly be feasible.

\section{Conclusions}

The C5-epimerization of D-glucuronic acid to its C5epimer L-iduronic acid has long been considered typical for animal derived glycosaminoglycans. However, an increasing number of L-iduronic acid containing structures in microorganisms can be confidently identified in prokaryotes as well. Moreover, we found multiple candidate Dglucuronyl C5-epimerases in a wide variety of microbes by in silico analysis of available prokaryotic genome data. Gene neighborhood analysis of these sequences suggests a role in sugar modification, most likely in type-specific polysaccharides (e.g. capsule polysaccharides or Oantigens). Phylogenetic analysis indicates sub-clustering of the set of candidate D-glucuronyl C5-epimerases into several clades. This possibly correlates with the existence of different C5-epimerase paralogs each having a distinct substrate specificity. The exact physiological function and substrate specificity requires biochemical analysis. However, this subset of sequences and Blast analysis of metagenomes reveal the existence of multiple candidate C5-epimerase genes in prokaryotes, supporting the conclusion that L-iduronic acid most likely is less rare in prokaryotes than expected. These putative C5epimerases potentially may become important tools in controlled chemo-enzymatic synthesis of L-iduronic acid containing polymers like heparin and heparin-analogs.
Acknowledgements We would like to thank Gijs van Dedem for critically reading the manuscript. This project is carried out in close collaboration with MSD Oss (formally Organon N.V.) and financially supported by the Netherlands Ministry of Economic Affairs and the BBasic partner organizations (www.b-basic.nl) through B-Basic, a public - private NWO-ACTS program (Advanced Chemical Technologies for Sustainability).

Open Access This article is distributed under the terms of the Creative Commons Attribution Noncommercial License which permits any noncommercial use, distribution, and reproduction in any medium, provided the original author(s) and source are credited.

\section{References}

1. Lindahl, U., Bäckström, G., Malmström, A., Fransson, L.A.: Biosynthesis of L-iduronic acid in heparin: Epimerization of Dglucuronic acid on the polymer level. Biochem. Biophys. Res. Commun. 46(2), 985-991 (1972)

2. Höök, M., Lindahl, U., Bäckström, G., Malmström, A., Fransson, L.A.: Biosynthesis of heparin. III. Formation of L-iduronic acid residues. J. Biol. Chem. 249, 3908-3915 (1974)

3. Valla, S., Li, J., Ertesvåg, H., Barbeyron, T., Lindahl, U.: Hexuronyl C5-epimerases in alginate and glycosaminoglycan biosynthesis. Biochimie 83(8), 819-830 (2001)

4. Lindahl, U.: 'Heparin'- from anticoagulant drug into the new biology. Glycoconj. J. 17(7-9), 597-605 (2000)

5. Ørskov, I., Ørskov, F., Jann, B., Jann, K.: Serology, chemistry, and genetics of $\mathrm{O}$ and $\mathrm{K}$ antigens of Escherichia coli. Bacteriol. Rev. 41(3), 667-710 (1977)

6. Rodriguez, M., Jann, B., Jann, K.: Structure and serological characteristics of the capsular K4 antigen of Escherichia coli O5: $\mathrm{K} 4$ : H4, a fructose-containing polysaccharide with a chondroitin backbone. Eur. J. Biochem. 177, 117-124 (1988)

7. Vann, W.F., Schmidt, M.A., Jann, B., Jann, K.: The structure of the capsular polysaccharide (K5 antigen) of urinary-tranctinfective Escherichia coli O10:K5:H4. A Polymer Similar to Desulfo-Heparin. Eur. J. Biochem. 116, 359-364 (1981)

8. Kusche, M., Hannesson, H.H., Lindahl, U.: Biosynthesis of heparin. Use of Escherichia coli K5 capsular polysaccharide as a model substrate in enzymic polymer-modification reactions. Biochem. J. 275, 151-158 (1991)

9. Hagner-McWhirter, A., Hannesson, H.H., Campbell, P., Westley, J., Rodén, L., Lindahl, U., Li, J.: Biosynthesis of heparin/heparan sulfate: kinetic studies of the glucuronyl $\mathrm{C} 5$-epimerase with $\mathrm{N}$-sulfated derivatives of the Escherichia coli K5 capsular polysaccharide as substrates. Glycobiology 10(2), 159-171 (2000)

10. Rimler, R.B.: Presumptive identification of Pasteurella multocida serogroups A, D and F by capsule depolymerisation with mucopolysaccharidases. Vet. Rec. 134, 191-192 (1994)

11. DeAngelis, P.L., Gunay, N.S., Toida, T., Mao, W., Linhardt, R.J.: Identification of the capsular polysaccharides of Type D and $\mathrm{F}$ Pasteurella multocida as unmodified heparin and chondroitin, respectively. Carbohydr. Res. 337(17), 1547-1552 (2002)

12. Rosner, H., Grimmecke, H.D., Knirel, Y.A., Shashkov, A.S.: Hyaluronic acid and a $(1 \rightarrow 4)-\beta$-D-xylan, extracellular polysaccharides of Pasteurella multocida (Carter type A) strain 880. Carbohydr. Res. 223, 329-333 (1992)

13. Darby, G.K., Jones, A.S., Kennedy, J.F., Walker, R.T.: Isolation and analysis of the nucleic acids and polysaccharides from Clostridium welchii. J. Bacteriol. 103(1), 159-165 (1970)

14. Lee, L., Cherniak, R.: Identification of iduronic acid as a constituent of the "type-specific" polysaccharide of Clostridium perfringens Hobbs 10. Carbohydr. Res. 33, 387-390 (1974) 
15. Stack, R.J., Plattner, R.D., Cote, G.L.: Identification of L-iduronic acid as a constituent of the major extracellular polysaccharide produced by Butyrivibrio fibrisolvens strain X6C61. FEMS Microbiol. Lett. 51, 1-5 (1988)

16. Hanniffy, O.M., Shashkov, A.S., Senchenkova, S.N., Tomshich, S.V., Komandrova, N.A., Romanenko, L.A., Knirel, Y.A., Savage, A.V.: Structure of a highly acidic O-specific polysaccharide of lipopolysaccharide of Pseudoalteromonas haloplanktis KMM 223 (44-1) containing L-iduronic acid and D-QuiNHb4NHb. Carbohydr. Res. 307, 291-298 (1998)

17. Nazarenkoa, E.L., Komandrovaa, N.A., Gorshkovaa, R.P., Tomshicha, S.V., Zubkova, V.A., Kilcoyneb, M., Savage, A.V.: Structures of polysaccharides and oligosaccharides of some Gram-negative marine Proteobacteria. Carbohydr. Res. 338(23), 2449-2457 (2003)

18. Perepelov, A.V., Liu, B., Senchenkova, S.N., Shashkov, A.S., Feng, L., Knirel, Y.A., Wang, L.: Structure of the Opolysaccharide of Escherichia coli O112ab containing Liduronic acid. Carbohydr. Res. 343(3), 571-575 (2008)

19. Liu, B., Knirel, Y.A., Feng, L., Perepelov, A.V., Senchenkova, S. N., Wang, Q., Reeves, P.R., Wang, L.: Structure and genetics of Shigella O antigens. FEMS Microbiol. Rev. 32, 627-653 (2008)

20. Wieland, F., Lechner, J., Sumper, M.: Iduronic acid: constituent of sulphated dolichyl phosphate oligosaccharides in halobacteria. FEBS Lett. 195, 77-81 (1986)

21. Wieland, F., Paul, G., Sumper, M.: Halobacterial flagellins are sulfated glycoproteins. J. Biol. Chem. 260(28), 15180-15185 (1985)

22. Jarrell, K.F., Bayley, D.P., Kostyukova, A.S.: The archaeal flagellum: a unique motility structure. J. Bacteriol. 178(17), 5057-5064 (1996)

23. Ozaki, N., Sakuda, S., Nagasawa, H.: A novel highly acidic polysaccharide with inhibitory activity on calcification from the calcified scale "coccolith" of a coccolithophorid alga, Pleurochrysis haptonemofera. Biochem. Biophys. Res. Commun. 357, 11721176 (2007)

24. Lahaye, M., Robic, A.: Structure and functional properties of ulvan, a polysaccharide from green seaweeds. Biomacromolecules 8, 1765-1774 (2007)

25. Miyasaki, T., Tsuchihashi, H., Yamada, H., Yodomae, T.: Detection and identification of L-iduronic acid in the glycuronan "protuberic acid" from Koboyasia nipponica. Carbohydr. Res. 77, 281-284 (1979)

26. Tsuchihashi, H., Yadomae, T., Miyazaki, T.: Structure of Lidurono-D-glucuronans from the fungi Aseroe araclmoidea and Pseudocolas fusiformis. Carbohydr. Res. 108(1), 123-128 (1983)

27. Carroll, A.R., Duffy, S., Avery, V.M.: Citronamides A and B, tetrapeptides from the Australian sponge Citronia astra. J. Nat. Prod. 72, 764-768 (2009)
28. Altschul, S.F., Madden, T.L., Schäffer, A.A., Zhang, J., Zhang, Z., Miller, W., Lipman, D.J.: Gapped BLAST and PSI-BLAST: a new generation of protein database search programs. Nucleic Acids Res. 25, 3389-3402 (1997)

29. Bateman, A., Coin, L., Durbin, R., Finn, R.D., Hollich, V., GriffithsJones, S., Khanna, A., Marshall, M., Moxon, S., Sonnhammer, E.L., Studholme, D.J., Yeats, C., Eddy, S.R.: The Pfam protein families database. Nucleic Acids Res. 32, D138-D141 (2004)

30. Rozeboom, H.J., Bjerkan, T.M., Kalk, K.H., Ertesvåg, H., Holtan, S., Aachmann, F.L., Valla, S., Dijkstra, B.W.: Structural and mutational characterization of the catalytic A-module of the Mannuronan C-5-epimerase AlgE4 from Azotobacter vinelandii. J. Biol. Chem. 283, 23819-23828 (2008)

31. Pacheco, B., Maccarana, M., Goodlett, D.R., Malmström, A., Malmström, L.: Identification of the active site of DS-epimerase 1 and requirement of $\mathrm{N}$-glycosylation for enzyme function. J. Biol. Chem. 284(3), 1741-1747 (2008)

32. Huson, D.H., Richter, D.C., Rausch, C., Dezulian, T., Franz, M., Rupp, R.: Dendroscope: An interactive viewer for large phylogenetic trees. BMC Bioinform. 8, 460 (2007)

33. Notredame, C., Higgins, D.G., Heringa, J.: T-Coffee: A novel method for multiple sequence alignments. J. Mol. Biol. 302, 205$217(2000)$

34. Thompson, J.D., Gibson, T.J., Plewniak, F., Jeanmougin, F., Higgins, D.G.: The ClustalX windows interface: flexible strategies for multiple sequence alignment aided by quality analysis tools. Nucleic Acids Res. 25, 4876-4882 (1997)

35. Whitfield, C.: Biosynthesis of lipopolysaccharide $\mathrm{O}$ antigens. Trends Microbiol. 3(5), 178-185 (1995)

36. Derzell, S., Turlin, E., Duchaud, E., Pages, S., Kunst, F., Givaudan, A., Danchin, A.: The PhoP-PhoQ two-component regulatory system of Photorhabdus luminescens is essential for virulence in insects. J. Bacteriol. 186(5), 1270-1279 (2004)

37. Reeves, P.R., Hobbs, M., Valvano, M.A., Skurnik, M., Whitfield, C., Coplin, D., Kido, N., Klena, J., Maskell, D., Raetz, C.R., Rick, P.D.: Bacterial polysaccharide synthesis and gene nomenclature. Trends Microbiol. 4(12), 495-503 (1996)

38. Chen, Y., Bystricky, P., Adeyeye, J., Panigrahi, P., Ali, A., Johnson, J.A., Bush, C.A., Morris Jr., J.G., Stine, O.C.: The capsule polysaccharide structure and biogenesis for non-O1 Vibrio cholerae NRT36S: genes are embedded in the LPS region. BMC Microbiol. 7, 20 (2007)

39. Bao, Q., Tian, Y., Li, W., Xu, Z., Xuan, Z., Hu, S., Dong, W., Yang, J., Chen, Y., Xue, Y., Xu, Y., Lai, X., Huang, L., Dong, X., Ma, Y., Ling, L., Tan, H., Chen, R., Wang, J., Yu, J., Yang, H.: A complete sequence of the $T$. tengcongensis genome. Genome Res. 12, 689-700 (2002) 